\title{
PSYCHOTIC SYMPTOMS AS A FIRST MANIFESTATION OF A MOSAIC TRISOMY 8
}

$19^{\text {TH }}$ WPA WORLD CONGRESS OF PSYCHIATRY LISBON, 21-24 AUGUST, 2019

\author{
L. Santos Silva, A. Batista, R. Araújo, M. Barbosa, J. Miranda, M. Duarte \\ Centro Hospitalar de Leiria-EPE, Serviço de Psiquiatria e Saúde Mental, Leiria, Portugal \\ luis.mds.silva@gmail.com
}

\section{Introduction:}

Mosaic trisomy 8 is a rare chromosomopathy, affecting males more often than females $(5: 1)$, whose clinical presentation has a wide phenotypic variability ranging from normal individual to severe malformations, that can affect multiple systems. Intellectual disability is the most common psychiatric feature, although some may have normal to borderline intelligence. There are few reports of psychotic symptoms in these patients; we intent to present a clinical report of a woman with trisomy 8 mosaicism that caught medical attention at first due to her psychiatric symptoms.

\section{Clinical Report:}

F. is a 23-year-old single woman, unemployed, with no relevant psychiatric history before she was 20 , nor psychiatric familiar history. She also had no significant medical history and had a normal psychomotor development. Her academic course was average, having finished the $12^{\text {th }}$ grade at 18 years old, with no disapprovals.

F. was first admitted at a psychiatric emergency service when she was 20 years-old. Her parents related that she was "different, saying and doing weird things"sic and presenting behavioural disturbances, for instance, leaving her house in the middle of the night, barefoot, and walk to the pinewood, climb up to trees.

She was initially evaluated by Internal Medicine colleagues. A slight dysmorphia of the hands and feet were noticed. A cranial CT scan and laboratorial tests were requested and no significant alterations were detected. At the psychiatric examination, F. was clearly disorganized, disoriented, with racing thoughts and expressing ideas of reference. She admitted auditory verbal hallucinations and sleep was also impaired. She had no insight about her condition. Despite her mother's objection, F. was admitted to the psychiatric inward.

A psychological assessment was requested and no major changes were detected, namely regarding her IQ. Drug abuse testing, serologies, vitamin deficiency and thyroid function tests were performed, with no abnormal results. After a few days, her sleep was restored and she had no psychotic symptoms, so she was discharged, medicated with Olanzapine $10 \mathrm{mg}$ id.

About a year later, F. was again admitted at the emergency department, after having interrupted her follow up appointments and medication. F. presented disorganized and incoherent speech, psychomotor retardation, thoughts blocking and loosening of associations. Her mood was depressed and her speech reflected the presence of delusions of guilt and suicidal thoughts "I hurt my brother, I don't know if I raped him, I will turn myself to the police, maybe I have to kill myself"sic.

During this second hospital stay, her recovery was very gradual and by the time of her discharge she still had some ideas of guilt. When she went back home, her psychiatric medication included: Olanzapine $10 \mathrm{mg}$ id, Aripiprazol $5 \mathrm{mg}$ id and Clomipramine $75 \mathrm{mg}$ id.
Her third hospitalization occurred at 23. At that time, she presented mental confusion, disorientation, loosening of associations, thoughts blocking and perplexity. According to her parents "she restarted saying she was going to go to jail because of what she had done" sic. F's behaviour did not get better; she showed signs of apathy and psychomotor retardation, making it difficult to maintain a conversation. A complete physical examination was performed and considering her dysmorphic hands/feet and short stature, x-ray exams were solicited.

X-ray results showed kyphoscoliosis and hypoplasia of the $3^{\text {rd }}, 4^{\text {th }}$ and $5^{\text {th }}$ metacarpals (Fig. 1). Given these results, her widely spaced nipples and short stature, the hypothesis of a genetic disorder was considered, resulting in a solicitation of a genetic study. About a month later, the karyotype (Fig. 2) confirmed the existence of a genetic disorder: Trisomy 8 mosaicism, also known as Warkany's syndrome - $(47, X X,+8 / 46, X X)$.

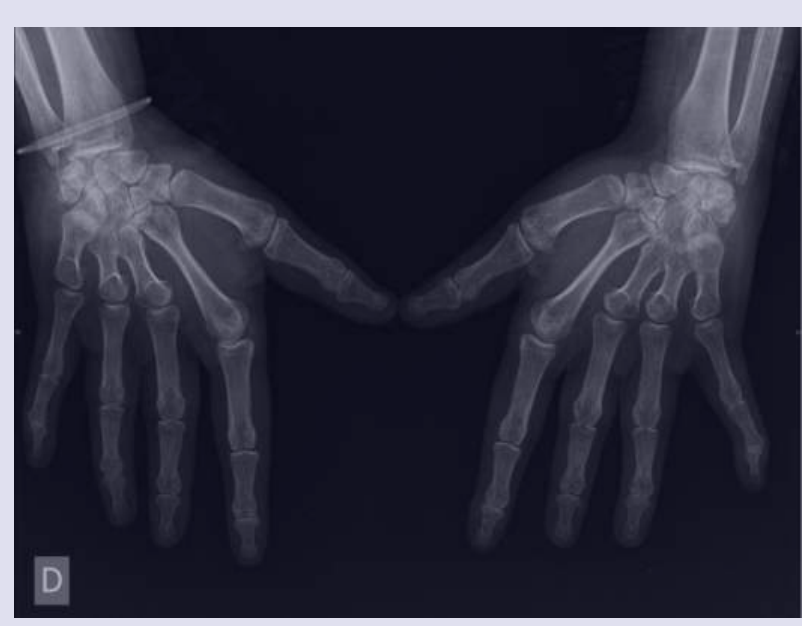

Fig. 1 - X-ray hands image

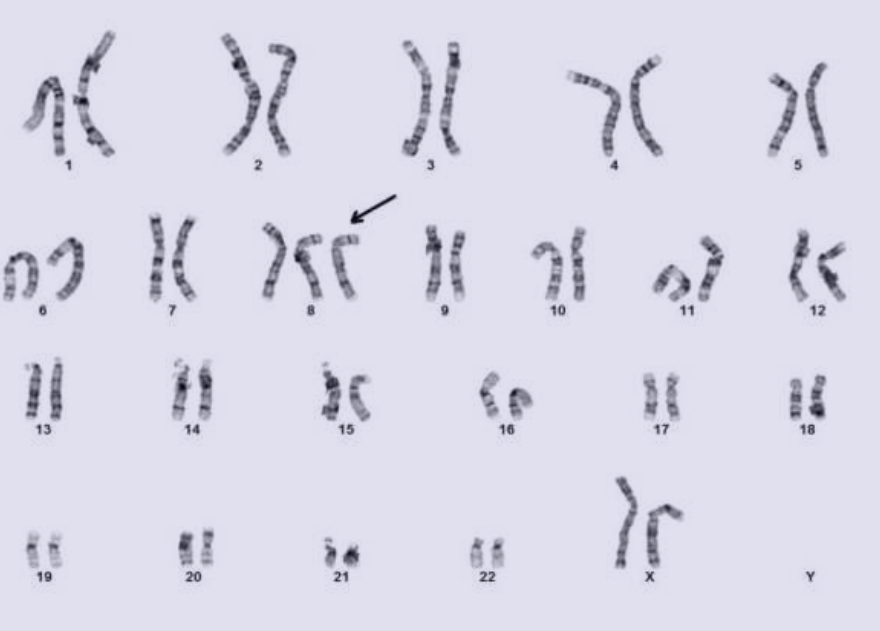

Fig. 2 - Karyotype (3 copies of chromosome 8)
Regarding psychiatric manifestations, mental retardation is the most reported abnormality. To our knowledge, concerning other psychiatric features, there are not many case reports.

In F's case, her psychotic symptoms are the most prominent ones, since they were the reason why she caught medical attention in the first place and led her to the subsequent investigation.

Unfortunately, after the diagnosis was confirmed, F's mother persuaded her to leave the inward facility, which led to her discharge. She was slightly better in terms of behaviour and thinking, although there was no marked improvement. When discharged, she was medicated with Risperidone $3 \mathrm{mg}$ id and Sertraline $100 \mathrm{mg}$ id.

\section{Discussion:}

Being a rare disease, with few available case reports, even more concerning the feminine gender, there are a lot of unanswered questions. The psychiatric diagnosis is still under consideration. F. is a girl who always had difficulties within social interaction; her history led us to hypothesize the possibility of an Autism spectrum disorder - a complete psychological evaluation should be performed, in order to clarify this possible diagnosis. F. also fulfills both ICD-10 and DSM5 criteria for schizophrenia, possibly a hebephrenic schizophrenia. She presented both delusions and auditory verbal hallucinations, but her disorganized thinking and behaviour were still the most prominent features of her clinical condition. Despite anti-depressive treatment, her affects were blunted, she lacked volition and she had a tendency for social isolation

Regarding psychiatric symptoms, it is still unknown if they are a part of the syndrome or a comorbidity, making it difficult to treat.

In this case, the slow progress and lack of improvement led to an extensive investigation led to the genetic diagnosis, otherwise she would have probably just had a psychiatric diagnosis and lacked her genetic follow up, seriously compromising her future. 